\title{
Idiopathic normal pressure hydrocephalus: diagnostic and predictive value of clinical testing, lumbar drainage, and CSF dynamics
}

\author{
"Cynthia V. Mahr, MD, ${ }^{1}$ Markus Dengl, MD, ${ }^{1}$ Ulf Nestler, MD, ${ }^{1}$ Martin Reiss-Zimmermann, MD, ${ }^{2}$ \\ Gerrit Eichner, ScD, ${ }^{3}$ Matthias Preuß, MD, ${ }^{1}$ and Jürgen Meixensberger, MD \\ ${ }^{1}$ Departments of ${ }^{1}$ Neurosurgery and ${ }^{2}$ Neuroradiology, University Hospital Leipzig; and ${ }^{3}$ Mathematical Institute, Justus-Liebig \\ University Gießen, Germany
}

\begin{abstract}
OBJECTIVE The aim of the study was to analyze the diagnostic and predictive values of clinical tests, CSF dynamics, and intracranial pulsatility tests, compared with external lumbar drainage (ELD), for shunt response in patients with idiopathic normal pressure hydrocephalus (iNPH).

METHODS Sixty-eight consecutive patients with suspected iNPH were prospectively evaluated. Preoperative assessment included clinical tests, overnight intracranial pressure (ICP) monitoring, lumbar infusion test (LIFT), and ELD for 24-72 hours. Simple and multiple linear regression analyses were conducted to identify predictive parameters concerning the outcome after shunt therapy.

RESULTS Positive response to ELD correctly predicted improvement after CSF diversion in $87.9 \%$ of the patients. A Mini-Mental State Examination (MMSE) value below 21 was associated with nonresponse after shunt insertion (specificity $93 \%$, sensitivity $67 \%$ ). Resistance to outflow of CSF (ROut) $>12 \mathrm{~mm} \mathrm{Hg} / \mathrm{ml} / \mathrm{min}$ was false negative in $21 \%$ of patients. Intracranial pulsatility parameters yielded different results in various parameters (correlation coefficient between pulse amplitude and ICP, slow wave amplitude, and mean ICP) but did not correlate to outcome. In multiple linear regression analysis, a calculation of presurgical MMSE versus the value after ELD, ROut, and ICP amplitude quotient during LIFT was significantly associated with outcome $(p=0.04)$.

CONCLUSIONS Despite a multitude of invasive tests, presurgical clinical testing and response to ELD yielded the best prediction for improvement of symptoms following surgery. The complication rate of invasive testing was $5.4 \%$. Multiple and simple linear regression analyses indicated that outcome can only be predicted by a combination of parameters, in accordance with a multifactorial pathogenesis of $\mathrm{NPH}$.
\end{abstract}

http://thejns.org/doi/abs/10.3171/2015.8.JNS151112

KEY WORDS normal pressure hydrocephalus; CSF dynamics; pulsatility

I DIOPATHIC normal pressure hydrocephalus (iNPH) has been a challenging entity for the past 50 years, since its first description by Hakim. ${ }^{21}$ Despite extensive research, its pathophysiological basis and pathogenesis remain matters of ongoing debate. Due to the uncertainty in diagnostic measures, several algorithms, diagnostic tools, and scoring systems have been proposed. A recent multicenter trial based the decision for surgical therapy on clinical signs and symptoms only, dividing cases into questionable and typical iNPH. ${ }^{23}$ Given the multitude of noninvasive and inva- sive measures to select symptomatic patients for hydrocephalus therapy, a prospective analysis of diagnostic and predictive parameters and their individual contributions within the diagnostic algorithm was warranted.

\section{Patients and Methods}

Patients with suspicious clinical and MRI signs for iNPH were examined in a prospective study. Local ethics committee approval was obtained.

ABBREVIATIONS AMP Q = ICP amplitude quotient; ELD = external lumbar drainage; ETV = endoscopic third ventriculostomy; ICP = intracranial pressure; iNPH = idiopathic normal pressure hydrocephalus; LIFT = lumbar infusion test; MMSE = Mini-Mental State Examination; mRS = modified Rankin Scale; OVM = overnight ICP monitoring; RAP = correlation coefficient between pulse amplitude and ICP; ROC = receiver operating characteristic; ROut = resistance to outflow of CSF; SLHS = Stein and Langfitt Hydrocephalus Score; VP = ventriculoperitoneal; $\Delta$ Kiefer = change in Kiefer score.

SUBMITTED May 19, 2015. ACCEPTED August 12, 2015.

INCLUDE WHEN CITING Published online January 29, 2016; DOI: 10.3171/2015.8.JNS151112.

* Drs. Mahr and Dengl contributed equally to this work. 
Seventy-four consecutive patients who presented with signs and symptoms of iNPH were identified clinically using the Kiefer score, Stein and Langfitt Hydrocephalus Score (SLHS), Mini-Mental State Examination (MMSE), standardized gait testing, grooved pegboard test, and modified Rankin Scale (mRS). ${ }^{4,7,8,10,15,19,20,23}$ In all patients, clinical evaluation prompted the indication for further invasive diagnostic examinations. Patients with a history of severe traumatic brain injury, previous CNS tumor surgery, cerebral infection, or hemorrhage were not included to avoid secondary normal pressure hydrocephalus. Six patients were excluded from the data analysis because testing was abandoned due to complications of invasive procedures $(n=4)$ or due to noncompliance $(n=2)$.

The diagnosis of iNPH was confirmed by a positive response to external lumbar drainage (ELD) in clinical testing together with positive self-assessment by the patient and relatives. ${ }^{14}$ Surgical therapy was offered to patients with a positive response only. According to the test results of ELD, patients were separated into 2 groups: those $(\mathrm{n}=$ 33 ) with a positive response to ELD (posELD) and those $(n=35)$ with a negative response to ELD (negELD). ELD served as the main clinical discrimination criterion during data analysis. Invasive testing included 3 examinations: 1) overnight intracranial pressure (ICP) monitoring (OVM) by parenchymal measurement for 24 to 48 hours, $2,5,22$ 2) standardized lumbar infusion test (LIFT), 4,6 and 3) ELD for 72 hours with pre- and postinterventional evaluation of the Kiefer score, MMSE, gait testing, and grooved pegboad test. ${ }^{4,6,10,14}$

Analysis of ICP and CSF dynamics studies were conducted with ICM+ software (Cambridge University Enterprise, Ltd.). ${ }^{17}$ The median correlation coefficient between pulse amplitude and ICP (RAP), amplitude of ICP pulse (1 harmonic sine wave), slow wave amplitude (using the morphological clustering and analysis of ICP pulse [MOCAIP] algorithm), and mean ICP values were recorded and calculated during OVM according to the protocols of Czosnyka et al. and Kasprowicz et al. . $^{2,3,9}$ LIFT yielded baseline and plateau values of ICP and pulse amplitudes as well as resistance of CSF outflow (ROut). Furthermore, the ICP amplitude quotient (AMP Q) was defined as the quotient between baseline and plateau ICP pulse wave amplitudes.

Clinical improvement of gait after ELD was defined as a $10 \%$ reduction of time and/or number of steps in a standardized gait test compared with pre-ELD testing. MMSE improvement was set at $10 \%$ increase of score points. Additionally, qualitative self-assessment by the patient and improvement reported by their relatives were taken into account.

iNPH patients were categorized as excellent responders (relief of all symptoms), improved patients (Kiefer score reduction of at least $10 \%$ ), and nonresponders (Kiefer score reduction of less than $10 \%)$. For prediction analysis of shunt response, excellent responders and the improved patients groups were merged as "responders." Furthermore, all patients were also categorized according to their LIFT results, into LIFT negative or positive subgroups; the test cutoff value for ROut was $12 \mathrm{~mm} \mathrm{Hg} / \mathrm{ml} / \mathrm{min}^{11,23}$

Thirty-three patients who underwent neurosurgical treatment were reevaluated 1 year after intervention. The primary outcome parameter was change in Kiefer score ( $\Delta$ Kiefer) values 12 months postoperatively. As intervention, ventriculoperitoneal (VP) shunts were suggested as the primary therapy for iNPH, and in 2 patients, endoscopic third ventriculostomy (ETV) was performed upon patient request.

If not stated otherwise, univariate 2-group comparisons and $\mathrm{p}$ values were calculated by Welch's 2 -tailed t-test in SPSS (version 20). The following 3 procedures were performed using the open-source software R: adjustment of $p$ values by Holm's correction method for multiple comparisons, a receiver operating characteristic (ROC) analysis for the parameter ROut, and multiple regression analyses for 5 models with $\Delta$ Kiefer as the dependent variable. The following 5 models comprised different sets of predictors that had a priori been proposed based on clinical experience and the literature and were considered to have a potential for outcome prediction: 1) ROut + preoperative MMSE + mean ICP of OVM + mean slow wave of OVM, 2) ROut + preoperative MMSE + AMP Q + MMSE change by ELD, 3) ROut + preoperative MMSE + AMP Q + gait test time change between pre- and post-ELD, 4) ROut + preoperative MMSE + AMP Q + gait test step number change between pre- and post-ELD, and 5) ROut + preoperative MMSE + AMP Q + mean amplitude of OVM.

All models were fitted with main effects and second order interaction effects. Significance level was set at 0.05 for all statistical analyses.

\section{Results}

Sixty-eight patients (47 males and 21 females) were evaluated. Mean age of the patients was 70.5 years (range 50-85 years, median 73 years). The mean duration between onset of the first symptoms and clinical testing was 15.2 months (range 1-48 months). The mRS and SLHS did not present any difference between the posELD and negELD groups. An overview of the preclinical test results of the patients is given in Table 1.

Positive ELD testing confirmed a diagnosis of iNPH (posELD) in 33 patients (48.5\%), and the diagnosis of iNPH was ruled out (negELD) in 35 patients (51.1\%). An overview of the preclinical test results is shown in Table 2. Thirty-one posELD patients were treated with a VPshunt system, whereas 2 iNPH patients opted for ETV as the primary therapy. One of the patients who underwent ETV did not benefit from the first surgery and received a shunt implantation in a second operation, which resulted in clinical improvement. A positive response to shunt therapy was found in 26 of the posELD patients (79\%) 12 months after therapy. Excellent responders and improved iNPH patients (collectively referred to as "responders") presented a similar mean $\Delta$ Kiefer of -4.8 and -4.2 , respectively (Table 3 ). The time course of improvement after VP-shunt surgery showed an increased reduction of Kiefer score after 4 weeks and a slight reduction of improvement until 12 months after surgery (Fig. 1). In all nonresponding patients, correct shunt function was positively tested to rule out insufficient drainage. An overview of the diagnostic power of individual tests and cutoff values is shown in Table 4. 
TABLE 1. Overview of preclinical assessment results in patient groups and subgroups before invasive diagnostic measurements during outpatient-based screening

\begin{tabular}{|c|c|c|c|c|c|c|c|c|c|c|}
\hline \multirow[b]{2}{*}{ Group \& Subgroup } & \multirow{2}{*}{$\begin{array}{l}\text { No. of } \\
\text { Pts }\end{array}$} & \multirow{2}{*}{$\begin{array}{l}\text { Mean Age } \\
\text { in Years }\end{array}$} & \multicolumn{4}{|c|}{ Mean Scores } & \multirow{2}{*}{$\begin{array}{c}\text { Mean GPB in } \\
\text { Seconds (\% NP) }\end{array}$} & \multirow{2}{*}{$\begin{array}{l}\text { Mean Gait Time in } \\
\text { Seconds (\% NP) }\end{array}$} & \multirow{2}{*}{$\begin{array}{c}\text { Mean } \\
\text { CMI }\end{array}$} & \multirow{2}{*}{$\begin{array}{l}\text { Mean Sx Duration } \\
\text { in Months }\end{array}$} \\
\hline & & & Kiefer & $m R S$ & SLHS & MMSE & & & & \\
\hline All & 68 & 70.5 & 6.7 & 3 & 2 & 25 & $172(15)$ & $13(16.0)$ & 2 & 16.4 \\
\hline NegELD & 35 & 72.9 & 6.7 & 3 & 2 & 25 & $178(17)$ & $12(11.4)$ & 2 & 14.1 \\
\hline PosELD & 33 & 70.3 & 7.1 & 3 & 2 & 26 & $182(12)$ & $15(30.3)$ & 3 & 17.9 \\
\hline Excellent & 11 & 68.8 & 5.1 & 2 & 1 & 28 & $128(0)$ & $18(18.2)$ & 2 & 18.5 \\
\hline Improved & 15 & 67.7 & 7.3 & 3 & 2 & 27 & $206(7)$ & $15(13.3)$ & 3 & 32.1 \\
\hline Nonresponder & 7 & 73.9 & 9.6 & 3 & 3 & 21 & $111(43)$ & $11(57.1)$ & 2 & 9.7 \\
\hline
\end{tabular}

$\mathrm{CMI}=$ comorbidity index; $\mathrm{GPB}=$ grooved pegboard test; NP = patients not able to perform test; $\mathrm{Pts}=$ patients; $\mathrm{Sx}=$ symptom.

\section{Diagnostic Value of Clinical Parameters}

Parameters to separate non-iNPH patients from iNPH patients are of highest clinical interest. Furthermore, parameters for shunt response prediction are of high practical importance in patient counseling and selection of patients for therapy.

Kiefer scores were not significantly different between posELD and negELD patients, but were slightly higher in nonresponders compared with responders. Among nonresponders a high percentage of patients $(57 \%)$ were not able to sufficiently perform gait testing due to inability to walk, and nonresponders had a higher prevalence of cognitive impairment (Table 1). We did not find significant differences in MMSE results between the ELD groups. Employing a cutoff value of 21 for the MMSE yielded a test specificity of $93 \%$ and a sensitivity of $67 \%$ to discriminate between responders and nonresponding patients (see Table 4). Preclinical gait testing for both time and step number did not disclose significant differences between responders and nonresponding patients $(\mathrm{p}=0.40)$.

\section{Diagnostic Value of Overnight ICP Monitoring}

OVM was performed in 64 patients. One patient declined implantation of the ICP probe, and measurement was incomplete in 3 patients due to dislocation of the ICP probe or technical problems. Mean ICP was not significantly different between negELD $(7.2 \mathrm{~mm} \mathrm{Hg})$ and iNPH patients $(9.8 \mathrm{~mm} \mathrm{Hg})(\mathrm{p}=0.12)$. Analysis of median RAP values showed a mean of 0.72 for negELD patients and $>$ 0.8 for shunt-responding patients (Fig. 2 and Table 2). Median RAP was not significantly different in comparisons between all subgroups of patients. Test specificity and sensitivity for RAP $>0.8$ in iNPH diagnostics were $70 \%$ and $74 \%$, respectively. Slow wave amplitudes showed no significant difference between the posELD and negELD groups or the shunt response groups. Mean ICP and slow wave values did not significantly differ between responders and nonresponders.

\section{Complications of Overnight ICP Monitoring}

Six patients of the initial cohort of 74 patients were

TABLE 2. Overview of test results for clinical and CSF dynamics of all tested patients*

\begin{tabular}{|c|c|c|c|c|c|c|c|c|c|c|}
\hline \multirow[b]{2}{*}{ Group \& Subgroup } & \multirow[b]{2}{*}{$\begin{array}{c}\text { No. of } \\
\text { Pts }\end{array}$} & \multicolumn{3}{|c|}{ Change Btwn Pre/Post-ELD Test (\%)† } & \multicolumn{4}{|c|}{ OVM } & \multicolumn{2}{|l|}{ LIFT } \\
\hline & & MMSE & GPB & $\begin{array}{l}\text { Gait Time } \\
\text { (seconds) }\end{array}$ & $\begin{array}{c}\text { ICP } \\
(\mathrm{mm} \mathrm{Hg})\end{array}$ & $\begin{array}{c}\text { Amp } \\
(\mathrm{mm} \mathrm{Hg})\end{array}$ & $\begin{array}{l}\text { Slow Wave } \\
(\mathrm{mm} \mathrm{Hg})\end{array}$ & $\begin{array}{c}\text { RAP } \\
\text { Median }\end{array}$ & $\begin{array}{l}\text { ROut (mm Hg/ } \\
\mathrm{ml} / \mathrm{min})\end{array}$ & Amp Q \\
\hline NegELD & 35 & +0.1 & +1.4 & +0.7 & 7.2 & 1.8 & 1.0 & 0.72 & 14 & 4.4 \\
\hline LIFT neg & 19 & -1.0 & +1.5 & +5.7 & 6.4 & 1.6 & 0.9 & 0.70 & 9 & 3.7 \\
\hline LIFT pos & 11 & +2.1 & -5.1 & -3.1 & 9.4 & 2.3 & 1.3 & 0.76 & 21 & 5.7 \\
\hline No LIFT & 5 & & & & & & & & & \\
\hline PosELD & 33 & -2.8 & -18.8 & -26.4 & 9.8 & 2.1 & 1.4 & 0.79 & 16 & 4.0 \\
\hline LIFT neg & 11 & -0.1 & +10.6 & -15.4 & 8.1 & 2.2 & 1.4 & 0.88 & 10 & 2.7 \\
\hline Lift pos & 15 & -3.6 & -12.5 & -30.6 & 10.3 & 2.0 & 1.4 & 0.78 & 18 & 4.4 \\
\hline No LIFT & 7 & & & & & & & & & \\
\hline Excellent & 11 & +0.8 & -39.0 & -34.1 & 9.6 & 1.9 & 1.4 & 0.85 & 17 & 3.8 \\
\hline Improved & 15 & +0.1 & -2.9 & -14.8 & 9.7 & 2.0 & 1.2 & 0.82 & 15 & 3.5 \\
\hline Nonresponder & 7 & -1.9 & +43.1 & -29.9 & 10.2 & 2.2 & 1.4 & 0.67 & 18 & 4.5 \\
\hline
\end{tabular}

* Values are means unless otherwise indicated; NegELD and PosELD represent patients with negative and positive response to 72 hours of ELD, respectively; LIFT negative and positive patients have been discriminated by a threshold of $12 \mathrm{~mm} \mathrm{Hg} / \mathrm{ml} / \mathrm{min}$; see Table 1 for percentage of patients not able to perform the gait test or GPB.

$\dagger$ Pre/post ELD evaluation shows relative changes in clinical test batteries before and after ELD for 72 hours.

‡ LIFT was not possible. 
TABLE 3. Overview of the neurological outcomes for all patients measured 12 months after surgical therapy compared to preoperative status

\begin{tabular}{|c|c|c|c|c|c|c|c|}
\hline \multirow[b]{2}{*}{ Group \& Subgroup } & \multirow[b]{2}{*}{ No. of Pts } & \multicolumn{6}{|c|}{ Change Btwn Preop \& 12 Months Postop* } \\
\hline & & MMSE $(\%)$ & GPB $(\%)$ & Gait Time (\%) & Gait Steps (\%) & Gait NE† & Kiefer \\
\hline All & 33 & +2.7 & -36.1 & -109 & -103.2 & 10 & -3.0 \\
\hline Excellent & 11 & +1.4 & -39.8 & -161.1 & -175.1 & 2 & -4.8 \\
\hline Improved & 15 & +4.8 & -18.4 & -62.9 & -39.4 & 2 & -4.2 \\
\hline Nonresponders & 7 & -42.3 & +14.9 & $-\ddagger$ & $-\ddagger$ & 6 & +0.8 \\
\hline
\end{tabular}

excluded from statistical analysis: 4 patients (5.4\%) were excluded during invasive testing due to complications, and 2 patients succumbed to severe nosocomial infections (1 pneumonia, 1 urosepsis) independent of the neurosurgical intervention. Of the 4 patients, 1 patient experienced a temporary neurological deficit from a small intracerebral hemorrhage after implantation of the ICP sensor (1.35\%). One patient suffered from a permanent severe hemiparesis despite prompt surgical evacuation of a major intracerebral hemorrhage $(1.35 \%)$. Testing was abandoned due to noncompliance in 2 patients.

\section{Diagnostic Value of the Lumbar Infusion Test}

A total of 60 LIFTs were conducted with simultaneous parenchymal ICP monitoring at a constant infusion rate of $1.5 \mathrm{ml} / \mathrm{min}$. In 8 patients, either implantation of lumbar drainage failed or the ICP probe became dislocated after OVM prior to lumbar infusion testing. Amplitude and ICP parameters did not yield any significant differences between the ELD groups. Specificity and sensitivity of the ROut test with a cutoff value of $12 \mathrm{~mm} \mathrm{Hg} / \mathrm{ml} / \mathrm{min}$ or higher ("positive LIFT result") were $51 \%$ and $79 \%$, respectively (Table 4). Mean ROut value of negELD patients was $14 \mathrm{~mm} \mathrm{Hg} / \mathrm{ml} / \mathrm{min}$ and, thus, was higher than the generally accepted cutoff value of $12 \mathrm{~mm} \mathrm{Hg} / \mathrm{ml} / \mathrm{min}^{2,4,11,23}$ Of 35 negELD patients, almost one-third showed a misleading positive LIFT result $(n=11)$.

Values of ROut in relation to treatment result $(\Delta$ Kiefer 12 months after surgery) are shown in Fig. 3. ROut values

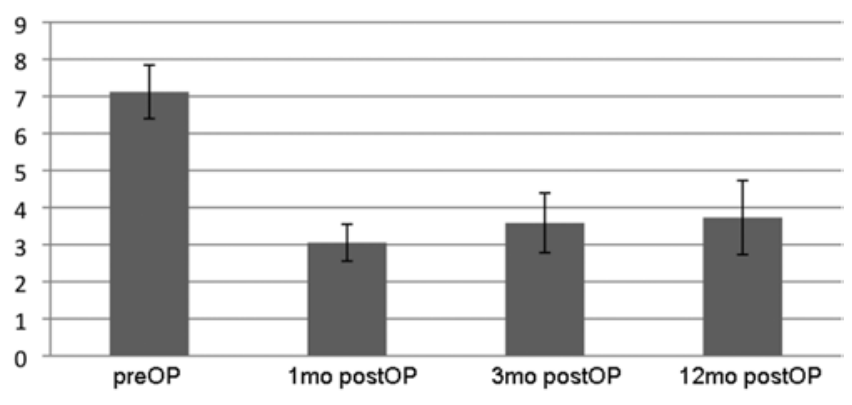

FIG. 1. Bar graph showing the time course of the mean modified Kiefer scores prior to surgery and 4 weeks, 3 months, and 12 months after surgery. were significantly different between negELD and posELD patients (Table 2). A ROC analysis of the lumbar infusion test is displayed in Fig. 4. The optimum cutoff for ROut for the present patient sample, based on the Youden index, was $13 \mathrm{~mm} \mathrm{Hg} / \mathrm{ml} / \mathrm{min} .{ }^{13}$

\section{Diagnostic Value of External Lumbar Drainage}

ELD was conducted in 65 patients for an average period of 66 hours, with a mean CSF flow of $6 \mathrm{ml} / \mathrm{hr}$, resulting in a mean CSF drainage volume of $445 \mathrm{ml}$ per patient (range 100-998 ml). Three patients underwent a high-volume tap test of 20-40 $\mathrm{ml}$ because ELD implantation was not possible due to spinal deformity or stenosis. Thirtytwo patients showed no change in clinical gait testing after ELD, and the results of gait testing in 3 patients worsened (negELD). In 1 patient, ELD had to be stopped after 12 hours due to massive deterioration of mental status. Subsequent cranial CT excluded intracranial complications of CSF withdrawal.

The differences in the results of gait testing and the MMSE after ELD did not reach statistical significance between shunt responding and nonresponding iNPH patients. Yet, a positive response to ELD correctly predicted improvement after shunt implantation in $29(87.9 \%)$ of the 33 patients with iNPH. Four patients (12.1\%) did not improve clinically after surgery, even though a positive effect had been found after ELD.

\section{Predictive Factors for Outcome After Shunt Therapy}

Univariate linear regression analysis of outcome $(\Delta$ Kiefer) and presurgical parameters did show a significant association with MMSE ( $p=0.043$ ) but not for gait testing $(\mathrm{p}=0.63)$. All OVM and LIFT parameters failed to result in significant relationships to outcome. Furthermore, 5 separate multiple regression models utilizing different combinations of diagnostic parameters of LIFT, OVM, and clinical parameters were analyzed for their ability to predict the shunt response of iNPH patients.

Of all models, only model 2 yielded a statistically significant relationship between $\Delta$ Kiefer and the input parameters.

Model 2 contained the predictor variables ROut, preoperative MMSE, AMP Q, and change in MMSE results between pre- and post-ELD. Using only the main effects 
TABLE 4. Overview of diagnostic power (sensitivity, specificity, negative and positive predictive value) of clinical parameters and different CSF dynamics parameters for shunt response*

\begin{tabular}{|c|c|c|c|c|c|c|c|c|c|c|c|}
\hline \multirow[b]{2}{*}{$\begin{array}{c}\text { Diagnostic } \\
\text { Power }\end{array}$} & \multicolumn{3}{|c|}{ Clinical Parameter } & \multicolumn{3}{|c|}{ OVM } & \multicolumn{4}{|c|}{ LIFT } & \multirow[b]{2}{*}{$\begin{array}{c}\text { ROut }>14+ \\
\text { RAP }>0.8\end{array}$} \\
\hline & $\begin{array}{c}\text { Kiefer } † \\
>5\end{array}$ & $\begin{array}{c}\text { Kiefer } † \\
>9\end{array}$ & $\begin{array}{c}\text { MMSE } \\
<21\end{array}$ & $\begin{array}{l}\text { RAP } \\
<0.7\end{array}$ & $\begin{array}{l}\text { RAP } \\
>0.8\end{array}$ & $\begin{array}{c}\text { Slow Wave } \\
>1.5\end{array}$ & $\begin{array}{c}\text { ROut } \\
>10\end{array}$ & $\begin{array}{c}\text { ROut } \\
>12\end{array}$ & $\begin{array}{c}\text { ROut } \\
>14\end{array}$ & $\begin{array}{c}\text { ROut } \\
>18\end{array}$ & \\
\hline Sensitivity & 58 & 4 & 93 & 91 & 74 & 35 & 96 & 79 & 63 & 25 & 35 \\
\hline Specificity & 71 & 43 & 67 & 41 & 70 & 71 & 31 & 51 & 63 & 83 & 84 \\
\hline PPV & 88 & 20 & 93 & 55 & 61 & 80 & 49 & 53 & 54 & 50 & 60 \\
\hline NPV & 31 & 11 & 67 & 86 & 81 & 25 & 92 & 78 & 71 & 62 & 65 \\
\hline
\end{tabular}

NPV = negative predictive value; $\mathrm{PPV}=$ positive predictive value.

* Values are \%; ROut unit is $\mathrm{mm} \mathrm{Hg} / \mathrm{ml} / \mathrm{min}$; slow wave amplitude unit is $\mathrm{mm} \mathrm{Hg}$.

† Global Kiefer score.

of these predictors, the model yielded a statistically significant relationship between the clinical and CSF-dynamics parameters and outcome $(p=0.046)$. Inclusion of all second order interactions led to a significantly improved model ( $\mathrm{p}=0.014)$, whose multiple $\mathrm{R}^{2}$ and adjusted $\mathrm{R}^{2}$ values were 0.79 and 0.60 , respectively. Second order interaction effects showed a clear ceiling effect for the parameters ROut and AMP Q at MMSE = 30. Interestingly, a significant negative association between ROut and $\Delta \mathrm{Kief}-$ er has been revealed: patients with high pathological ROut values showed less improvement in $\Delta$ Kiefer than patients with normal or low ROut values $(\mathrm{p}=0.039)$.

In summary, there is a statistically significant contribution of preoperative MMSE value and MMSE change after ELD as well as ROut and AMP Q during LIFT as covariables to the prediction of outcome. This corresponds with the results of univariate analysis of preoperative MMSE differences between the subgroups of positive or negative shunt-response depending on a critical MMSE threshold of 21 .

\section{Discussion}

In the present study, 68 symptomatic patients were evaluated for the presence of iNPH by clinical and invasive tests. Nonresponders to shunt therapy had a signifi- cantly decreased mental performance in MMSE (mean value 20.8), included a high percentage of severe walkingimpaired patients (57\%), and suffered from an elevated Kiefer score (mean 9.6). Conversely, MMSE values below 21 seem to make patients prone to nonresponse (80\% nonresponders in that subgroup of patients) to shunt therapy. Alternatively, severe cognitive impairment measured by MMSE may also indicate that patients suffer from neurological comorbidities or other entities mimicking iNPH. Potential differential diagnoses such as dementia or Alzheimer's disease should be considered when counseling these patients.

Shunt response resulted in an average improvement ( $\Delta$ Kiefer of about -4.1 ), without a big difference between excellent neurological result or "merely" improved patients. Excellent responders had been less severely affected by a lower presurgical Kiefer score of 5.1 versus 7.3 in improved iNPH patients. These data suggest that treatment of hydrocephalus with severe impairment increases the risk of therapy failure due to a "point-of-no-return," as postulated by previous authors., 16 Our findings indirectly support assumptions in which metabolic disturbances add to pulsation disturbances in iNPH during progression of the condition. ${ }^{1,15,23}$ However, it was not possible to identify an association between duration of symptoms and presur-
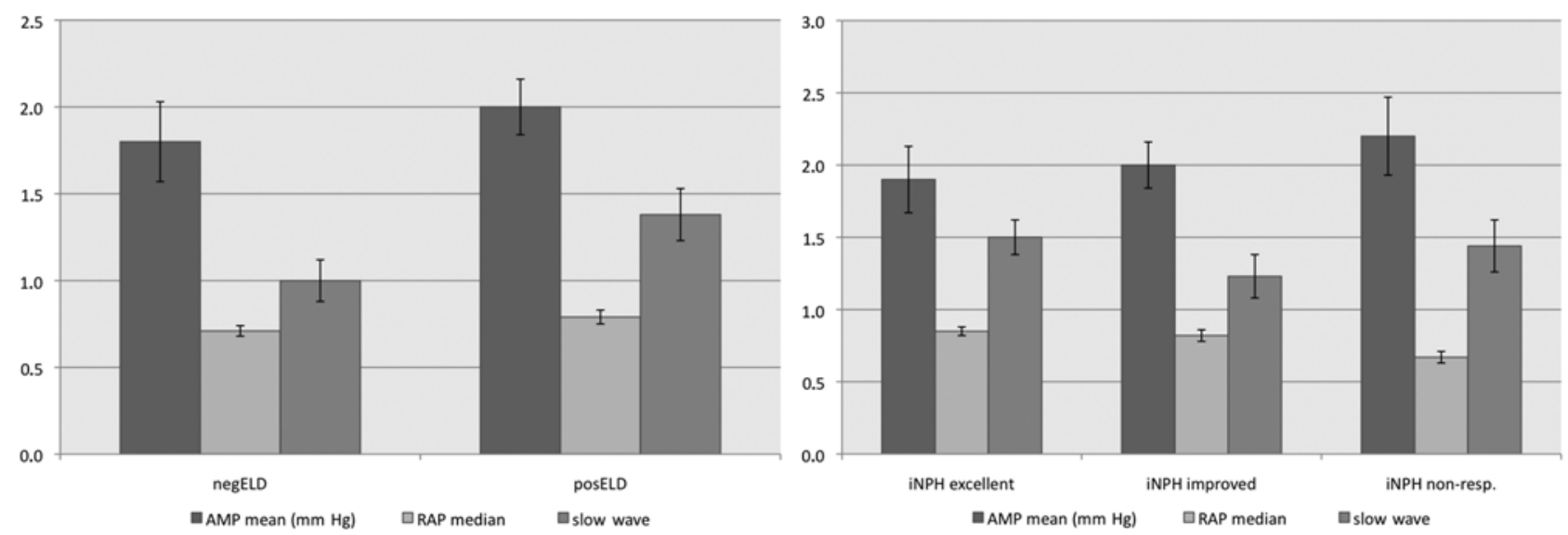

FIG. 2. Bar graph showing the results of the OVM pulsatility analysis. Left: Groups of positive (posELD) and negative (negELD) responders to ELD. Right: Results of patient groups according to their response 12 months after shunt therapy. 


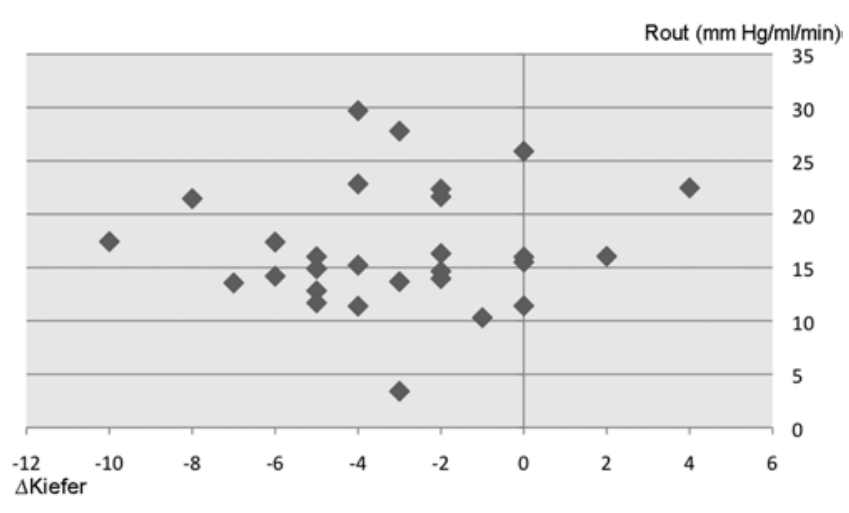

FIG. 3. Scatter plot showing the correlation between outcome ( $\Delta$ Kiefer 12 months after surgery) and ROut values of preoperative LIFT.

gical Kiefer scores (i.e., severity of symptoms) in our study sample.

In this preselected symptomatic group of patients, positive predictive value and negative predictive value for ROut $>12 \mathrm{~mm} \mathrm{Hg} / \mathrm{ml} / \mathrm{min}$ were $53 \%$ and $78 \%$, respectively. Thus, in our hands, nearly one half of the LIFT predictions based on ROut would have been false positive. Therefore, LIFT is not an ideal test to exclude patients from treatment, which is in concordance with the results of a recent multicenter trial. ${ }^{23}$ The LIFT false-positive patients, without response to shunt therapy but with intolerance to CSF infusion, constitute a group who requires further investigation for hydrocephalic pathophysiology and possible treatment options. ${ }^{18}$ In accordance, ROut values did not show a linear correlation to $\Delta$ Kiefer $\left(R^{2}=0.03\right)$ (Fig. 3).

In summary, neither one of the OVM or LIFT parameters nor a combination of them proved to be superior to clinical examination and ELD in regards of diagnostic power (Table 4).

Predictive parameters for iNPH treatment have a high clinical importance for patient counseling but have not yet been identified. ${ }^{13,19,22,23}$ In the present study, multiple regression analysis disclosed a relation between preoperative MMSE, cognitive change after ELD, and the outcome after shunt therapy. Patients with an MMSE below 21 and/ or worsening of cognitive function after ELD should be regarded as high risk for nonresponse to shunt therapy, especially when ROut measurements show pathological values above the cutoff of $14 \mathrm{~mm} \mathrm{Hg} / \mathrm{ml} / \mathrm{min}$.

Despite several statistical differences of CSF dynamics measured by OVM and LIFT for diagnostic purposes, a single predictive parameter cannot be derived from the

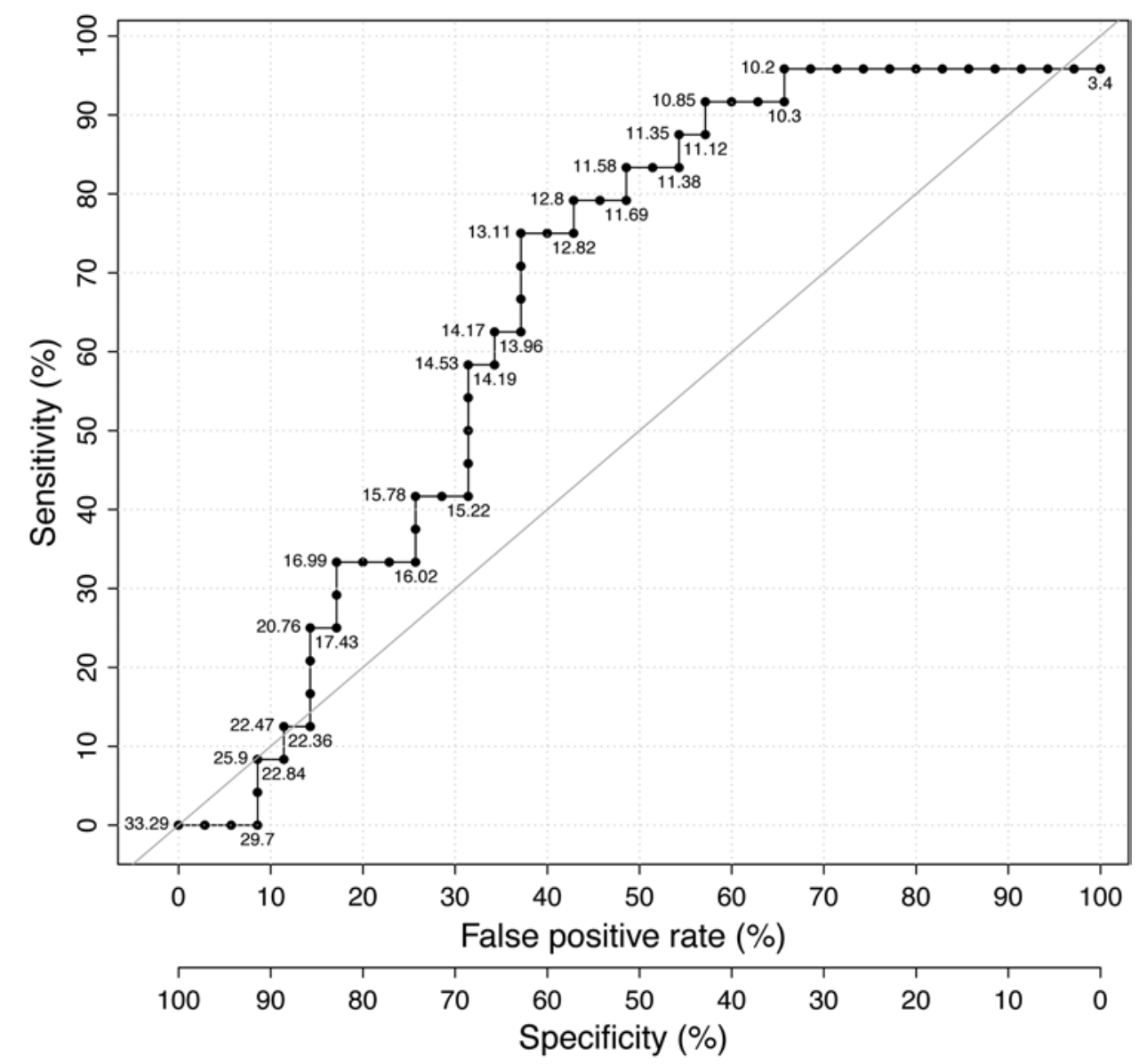

FIG. 4. Plot of the ROC analysis of ROut values after LIFT for the discrimination between posELD and negELD patients. The optimum cutoff value according to the Youden index is at $13 \mathrm{~mm} \mathrm{Hg} / \mathrm{ml} / \mathrm{min}$, with a specificity of $63 \%$ and sensitivity of $75 \%$. 
invasive procedures for the individual patient. These results support the hydrocephalus theory of a multifaceted condition of hydraulic, mechanical, and metabolic disorders as well as counter-regulatory mechanisms that may secondarily lead to impaired neuronal function and damage. $1,12,15,18$

\section{Conclusions}

In this study, ELD together with standardized clinical testing were reliable positive selection criteria for shunt response in iNPH, with a correct prediction in $87.9 \%$ of the treated patients. In our hands, ELD proved to be safe and was associated with fewer complications than other invasive measurements of ICP. In light of an overall invasive diagnostic complication rate of 5.4\%, LIFT and OVM should be reserved for questionable iNPH patients to add further diagnostic details. Patients with MMSE below 21 or cognitive worsening during ELD have a high probability for nonresponse after neurosurgical CSF diversion.

\section{References}

1. Andrén K, Wikkels $\varnothing$ C, Tisell M, Hellström P: Natural course of idiopathic normal pressure hydrocephalus. J Neurol Neurosurg Psychiatry 85:806-810, 2014

2. Czosnyka M, Czosnyka Z, Keong N, Lavinio A, Smielewski $\mathrm{P}$, Momjian S, et al: Pulse pressure waveform in hydrocephalus: what it is and what it isn't. Neurosurg Focus 22(4):E2, 2007

3. Czosnyka M, Smielewski P, Timofeev I, Lavinio A, Guazzo E, Hutchinson P, et al: Intracranial pressure: more than a number. Neurosurg Focus 22(5):E10, 2007

4. Czosnyka Z, Czosnyka M, Owler B, Momjian S, Kasprowicz M, Schmidt EA, et al: Clinical testing of CSF circulation in hydrocephalus. Acta Neurochir Suppl 95:247-251, 2005

5. Czosnyka Z, Keong N, Kim DJ, Radolovich D, Smielewski $\mathrm{P}$, Lavinio A, et al: Pulse amplitude of intracranial pressure waveform in hydrocephalus. Acta Neurochir Suppl 102:137-140, 2008

6. Eide PK, Brean A: Cerebrospinal fluid pulse pressure amplitude during lumbar infusion in idiopathic normal pressure hydrocephalus can predict response to shunting. Cerebrospinal Fluid Res 7:5, 2010

7. Farrell B, Godwin J, Richards S, Warlow C: The United Kingdom transient ischaemic attack (UK-TIA) aspirin trial: final results. J Neurol Neurosurg Psychiatry 54:1044-1054, 1991

8. Folstein MF, Folstein SE, McHugh PR: "Mini-mental state". A practical method for grading the cognitive state of patients for the clinician. J Psychiatr Res 12:189-198, 1975

9. Kasprowicz M, Asgari S, Bergsneider M, Czosnyka M, Hamilton R, Hu X: Pattern recognition of overnight intracranial pressure slow waves using morphological features of intracranial pressure pulse. J Neurosci Methods 190:310-318, 2010

10. Kiefer M, Eymann R, Komenda Y, Steudel WI: [A grading system for chronic hydrocephalus.] Zentralbl Neurochir 64:109-115, 2003 (Ger)

11. Kim DJ, Kim H, Kim YT, Yoon BC, Czosnyka Z, Park KW, et al: Thresholds of resistance to CSF outflow in predicting shunt responsiveness. Neurol Res 37:332-340, 2015
12. Kondziella D, Sonnewald U, Tullberg M, Wikkelso C: Brain metabolism in adult chronic hydrocephalus. J Neurochem 106:1515-1524, 2008

13. López-Ratón M, Rodriguez- Á lvarez MX, Cadarso-Suárez C, Sampedro FG: OptimalCutpoints: An R package for selecting optimal cutpoints in diagnostic tests. J Stat Softw 61(8): 1-36, 2014

14. Panagiotopoulos V, Konstantinou D, Kalogeropoulos A, Maraziotis T: The predictive value of external continuous lumbar drainage, with cerebrospinal fluid outflow controlled by medium pressure valve, in normal pressure hydrocephalus. Acta Neurochir (Wien) 147:953-958, 2005

15. Preuss M, Hoffmann KT, Reiss-Zimmermann M, Hirsch W, Merkenschlager A, Meixensberger J, et al: Updated physiology and pathophysiology of CSF circulation - the pulsatile vector theory. Childs Nerv Syst 29:1811-1825, 2013

16. Rankin J: Cerebral vascular accidents in patients over the age of 60. II. Prognosis. Scott Med J 2:200-215, 1957

17. Smielewski P, Czosnyka Z, Kasprowicz M, Pickard JD, Czosnyka M: ICM+: a versatile software for assessment of CSF dynamics. Acta Neurochir Suppl 114:75-79, 2012

18. Speil A, Sosa JC, Will BE, Schuhmann MU: Lack of correlation of overnight monitoring data and lumbar infusion data in iNPH patients. Acta Neurochir Suppl 114:213-216, 2012

19. Stein SC, Langfitt TW: Normal-pressure hydrocephalus. Predicting the results of cerebrospinal fluid shunting. J Neurosurg 41:463-470, 1974

20. Tanaka N, Yamaguchi S, Ishikawa H, Ishii H, Meguro K: Prevalence of possible idiopathic normal-pressure hydrocephalus in Japan: the Osaki-Tajiri project. Neuroepidemiology 32:171-175, 2009

21. Wallenstein MB, McKhann GM II: Salomón Hakim and the discovery of normal-pressure hydrocephalus. Neurosurgery 67:155-159, 2010

22. Weerakkody RA, Czosnyka M, Schuhmann MU, Schmidt E, Keong N, Santarius T, et al: Clinical assessment of cerebrospinal fluid dynamics in hydrocephalus. Guide to interpretation based on observational study. Acta Neurol Scand 124:85-98, 2011

23. Wikkelsø C, Hellström P, Klinge PM, Tans JT: The European iNPH Multicentre Study on the predictive values of resistance to CSF outflow and the CSF Tap Test in patients with idiopathic normal pressure hydrocephalus. J Neurol Neurosurg Psychiatry 84:562-568, 2013

\section{Disclosures}

This study was funded by B. Braun Aesculap AG.

\section{Author Contributions}

Conception and design: Dengl, Meixensberger. Acquisition of data: Preuß, Mahr, Dengl. Analysis and interpretation of data: Preuß, Mahr, Reiss-Zimmermann, Eichner. Drafting the article: Mahr. Critically revising the article: Preuß, Nestler, Meixensberger. Reviewed submitted version of manuscript: Dengl, Nestler, Reiss-Zimmermann, Eichner. Statistical analysis: Mahr, Dengl, Eichner. Administrative/technical/material support: Meixensberger.

\section{Correspondence}

Matthias Preuß, Department of Neurosurgery, University Hospital Leipzig, Liebigstrasse 20, 04103 Leipzig, Germany. email: preuss@kinderneurochirurgie-leipzig.de. 